\title{
EVAULATION OF A MODEL FOR URBAN VEGETATION BARRIERS' EFFECTS ON AIR POLLUTION
}

Ray F. Nassar, Nicolas Carrasco, Roger Rushworth, Charles O'Donnell. Engineering and Science University Magnet School, 500 Boston Post Road, West Haven, CT, USA

\begin{abstract}
Air pollution is a serious global issue, responsible for approximately one in every nine deaths each year, ranking it among the greatest environmental hazards to human health. It is of particular concern in urban areas, where elevated pollutant concentrations and potential sufferers converge. Over one half of the world's population presently lives in urban areas, and the urban population ratio is expected to reach $68 \%$ by 2050 . Common air pollutants include particulate matter (PM), sulphur dioxide (SO2), ground-level ozone (O3), nitrogen oxide (NOx) and carbon monoxide $(\mathrm{CO})$. While elevated rates of air pollution pose serious health risks for humans, outdoor plants can help reduce the harmful effects of air pollution by filtering and purifying the air around us.

In this project Common Ivy, Aster and Miniature Andromeda plants were evaluated for air pollutant mitigation. In this study we developed a vegetation barrier model with the plant located in the middle of the greenhouse box, and air pollutant was sprayed on one side of the plant. Dispersion patterns of sprayed pollutants were tested with and without vegetation barrier. Measurements of carbon dioxide (CO2), Formaldehyde ( $\mathrm{HCHO}$ ), Total Volatile Organic Compounds (TVOC), and Particulate Matter (PM2.5/PM10) were taken before spraying, then at 0 and 30 minutes after spraying, using both monitors.

The results show mitigation rates (in $177 \mathrm{ft} 3$ of air after $30 \mathrm{~min}$ ): for TVOC the minimum reduction is $5 \mathrm{mg} / \mathrm{m} 3$; for $\mathrm{HCHO}, 1 \mathrm{mg} / \mathrm{m} 3$; for CO2, $2000 \mathrm{ppm}$; for PM2.5, $2000 \mathrm{ug} / \mathrm{m} 3$; and for PM10 it was $1000 \mathrm{ug} / \mathrm{m} 3$.
\end{abstract}

\section{HYPOTHESIS}

Polluted air contains particles, odors and harmful gasses like nitrogen oxides, sulfur dioxide and ammonia. Green infrastructure (i.e., vegetation) can play a significant role in mitigating urban air pollution both by actual reduction in pollutant levels and by serving as a barrier 
between sources and exposed populations. As pollutants settle on the leaves of trees and plants, or are absorbed through their stomata (pores), the plants filter these harmful substances from the air.

The hypothesis: plants can be used as barriers from harmful pollutants in air, as well as significant mitigants. Using plants with larger surface area per leaf may mitigate a substantial amount of pollutants.
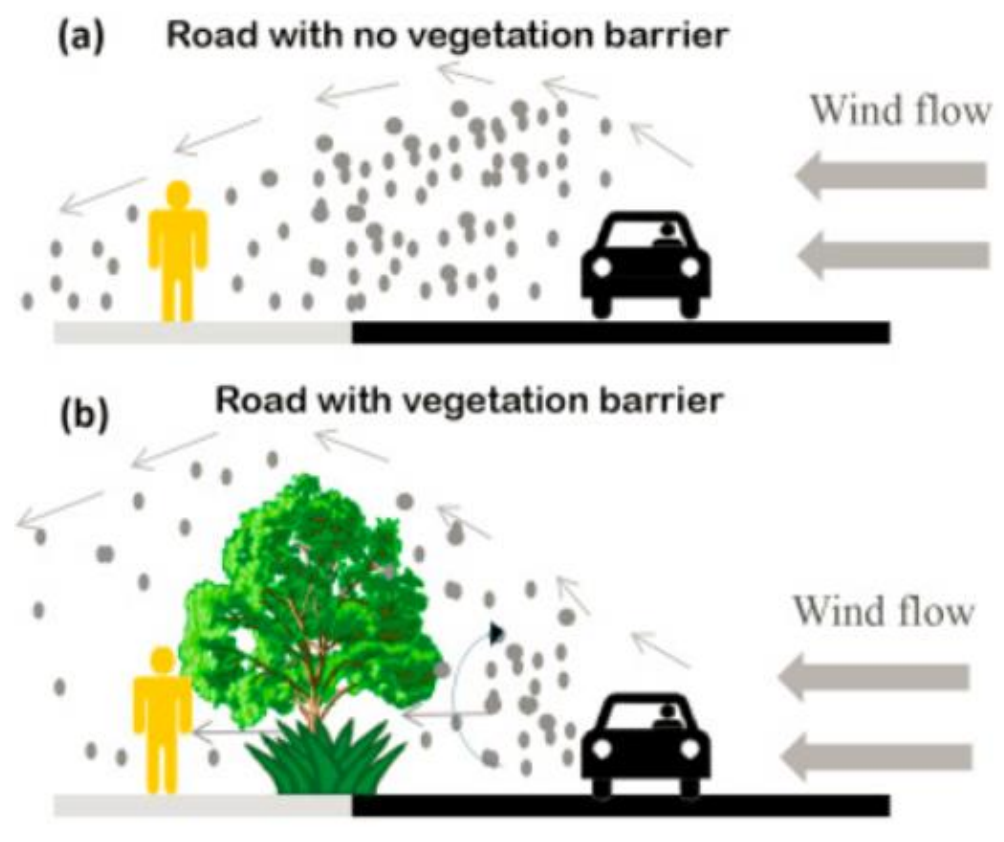

Figure 1. Dispersion patterns of road pollutants under open road configurations (a) without vegetation barrier and (b) with vegetation. (Adapted from ref 5)

\section{INTRODUCTION}

A green environment can provide the cleanest and safest place for humans to live. Trees and plants are oxygen generating factories that cleanse air and absorb carbon dioxide, thus improving the quality of the air for animals and humans to breathe. It has been shown that the amount of air pollution which is removed in leaf season, during daytime, is typically less than $1 \%$ but can be as high as $16 \%$ in areas with dense trees (1). For combating a variety of respiratory issues and other illnesses caused by air pollution, there can be no better way than planting some chosen varieties of plants that can cleanse the air and make our environment better. It is widely 
understood that urban vegetation has a natural ability to reduce air pollution and help to reduce the effects of climate change induced by rising temperatures.

The relationship between vegetation and reduction of population exposure to air pollution in urban areas has been investigated (2). The need for such research is intensified by projected global population growth, which is increasingly concentrated in urban settings, along with the effects of climate change on local, regional and worldwide weather patterns and overall atmospheric conditions. Transportation emissions are a major source of urban air pollution, and are characterized by a range of noxious gaseous pollutants, such as carbon monoxide (CO) and nitrogen oxides $\left(\mathrm{NO}_{\mathrm{x}}\right)$, along with particulate matter (PM), including black carbon (BC). Beyond vehicle exhaust emissions, non-exhaust but traffic-related emissions (e.g. particulate matter resulting from brake wear) are considered to be significant sources of PM, which is then resuspended by traffic-related turbulence.

Through the use of carefully selected vegetation to form roadside barriers, it is possible to reduce pedestrian exposure to air pollution. Polluted air is directed by wind either over or through the vegetation barriers; this is dependent upon porosity and physical dimensions. Trees remove gaseous air pollution primarily by uptake via leaf stomata, though some gases are removed by the plant surface (3). Trees also remove pollution by intercepting airborne particles. The intercepted particle often is resuspended to the atmosphere, washed off by rain, or dropped to the ground with leaf and twig fall. In this study we developed a vegetation barrier model with the plant located in the middle of the greenhouse box, and air pollutant was sprayed on one side of the plant. Dispersion patterns of sprayed pollutants were tested with and without vegetation barrier.

\section{METHOD}

Materials. Air Quality Monitor: Yvelines Air Quality Tester for CO2, Formaldehyde (HCHO), TVOC, PM2.5/PM10 was used, with settings for Multifunctional Air Gas Detector Real Time Data \& Mean Value Recording. It uses electrochemical sensors that can accurately detect the presence of Carbon Dioxide (CO2), Formaldehyde (HCHO), Total Volatile Organic Compound (TVOC), and Particulate Matter (PM2.5/PM10). Green House Box: MCombo Double Box Wooden Greenhouse Cold Frame Raised Plants Bed Protection, overall dimension:35.4"x31.3"x23.0"(LXWXH) (177 ft3) was used. Spray: Watco - Gloss Lacquer Solvent Based Aerosol, which contains high concentrations of n-butyl acetate, liquid petroleum 
gas, acetone, 2-methyl propyl, isobutyrate, isopropanol, methyl ethyl ketone, nitrocellulose, and toluene.

Plants (Figure 2). Common Ivy plant: this plant has leaves with a large surface area, making it an ideal choice for air purification, as it produces a good amount of oxygen, thereby freshening the air. Aster plant: this plant is known as a bioindicator plant, one that can change its appearance when the surrounding air is polluted beyond a particular level. Thus we can observe that the air quality is deteriorating and take the necessary steps to protect ourselves. Miniature Andromeda: A simply stunning dwarf selection with a unique columnar habit. Clusters of white flowers cover the plant in early spring. Miniature foliage emerges chartreuse before maturing to glossy green. Its unique compact stature makes it a perfect choice for smaller gardens and tight urban spaces.

Procedure. Each of the three plants was placed in the center of the greenhouse box, with air quality monitors placed on either side, as shown in Figure 3. The monitors were turned on, and measurements taken for CO2, Formaldehyde (HCHO), TVOC, and PM 2.5/PM10. Next, the aerosol pollutant spray was introduced at the right end of the greenhouse box, and measurements were taken at 0 min. for the above-listed pollutants. A second set of readings was taken after 30 min. As a control, the spray was introduced into the box with no plant present.

\section{RESULTS AND DISCUSSION}

Quality assurance and control. We first performed a control experiment without any of the plants in the green house box. A consistent quality assurance approach was maintained by testing the Yvelines Air Quality Tester for CO2 Formaldehyde (HCHO), TVOC, PM2.5/PM10 in a clean environment and recalibrating to zero before each experiment. Also the experiment was repeated three times for the control and each of the plants and we then took the average.

Miniature Andromeda, Aster and Common Ivy plants were evaluated for their air pollutant mitigation properties. As Figure 4 indicates, the results show that, in $177 \mathrm{ft} 3$ of air in 30 minutes, the minimum amounts of TVOC which the plants mitigated were $5 \mathrm{mg} / \mathrm{m} 3$; and the minimum amount of $\mathrm{HCHO}$ in the same time period is more than $1 \mathrm{mg} / \mathrm{m} 3$. Figure 5 shows that the plants mitigated at least 2000 ppm of CO2 in 30 minutes; in addition, the amounts of PM2.5 are more than $2000 \mathrm{ug} / \mathrm{m} 3$, and the amounts of PM10 which was mitigated are more than $1000 \mathrm{ug} / \mathrm{m} 3$. 
Vegetation barriers. Rows of planted trees and other vegetation types provide a barrier between the road and population groups in adjacent residential areas. Vegetation barriers force polluted air either over or through the vegetation; this is dependent upon porosity and physical dimensions. The leaves and plant surface absorb these pollutants through their stomata (pores) and filter these harmful substances from the air. Urban air pollution can be further mitigated through planning and design strategies, including vegetation preservation and planting, building design and development, installation of roadside and near-source structures, and modification of surrounding terrain features.

\section{CONCLUSIONS}

- Model for testing effects of vegetation barriers on urban air pollution was developed

- Outdoor plants can help beat the detrimental effects of air pollution and purify the air around us.

- Vegetation in urban settings can provide numerous benefits beyond air quality improvements, including temperature and storm water regulation, noise reduction, aesthetic improvements, and environments conducive to physical exercise and experiencing nature.

\section{REFERENCES}

1. David J. Nowak, Daniel E. Crane, Jack C. Stevens. Air pollution removal by urban trees and shrubs in the United States. Urban Forestry \& Urban Greening 4 (2006) 11 5-123

2. Richard William Baldauf, David J. Nowak. Vegetation and Other Development Options for Mitigating Urban Air Pollution Impacts. In book: Global Environmental Change (pp.479-485)

3. Smith, William H. Air Pollution and Forests. Interactions between Air Contaminants and Forest Ecosystems, 1990, Springer, NewYork.

4. C. Nick Hewitt, Kirsti Ashworth \& A. Rob MacKenzie. Using green infrastructure to improve urban air quality (GI4AQ). Ambio volume 49, pages62-73(2020).

5. K.V. Abhijitha, et al. Air pollution abatement performances of green infrastructure in openroad and built-up street canyon environmentse. A review. Atmospheric Environment, Volume 162, August 2017, Pages 71-86 


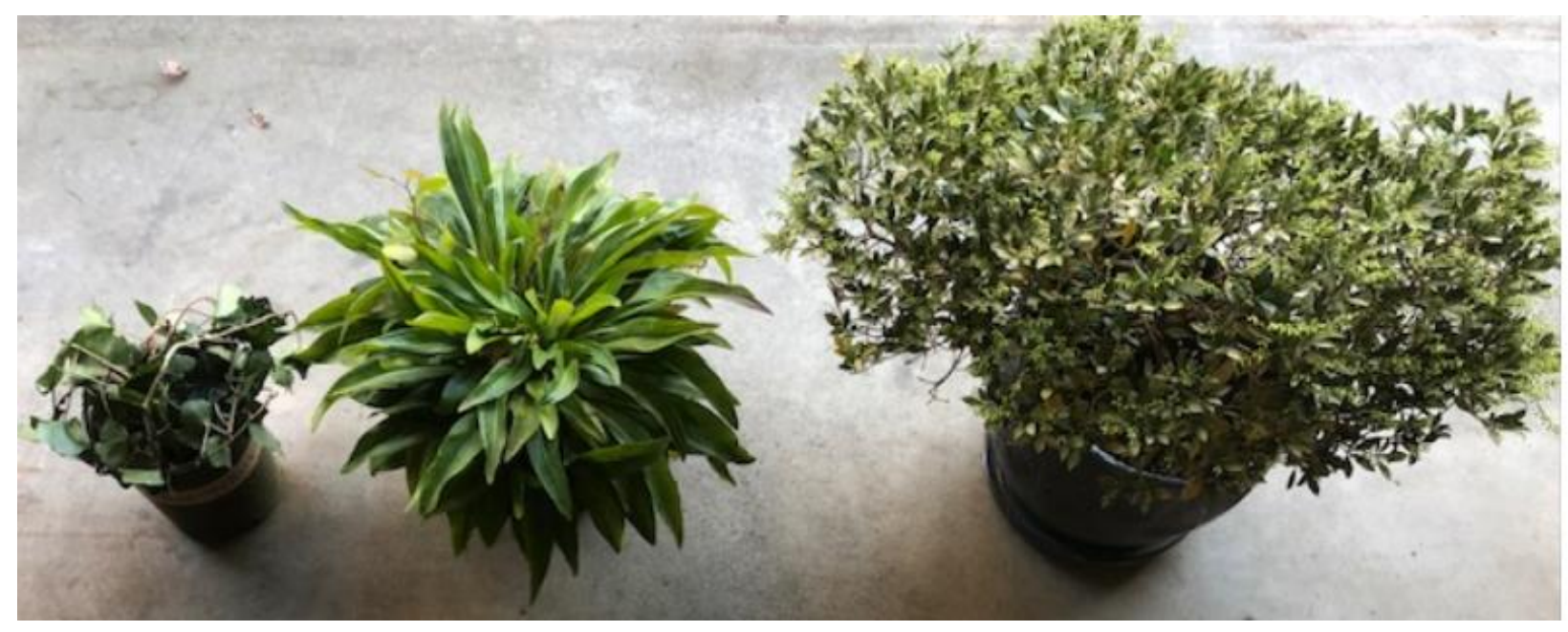

Figure 2. Plants from left to right Common Ivy plant, Aster plant and Miniature Andromeda 


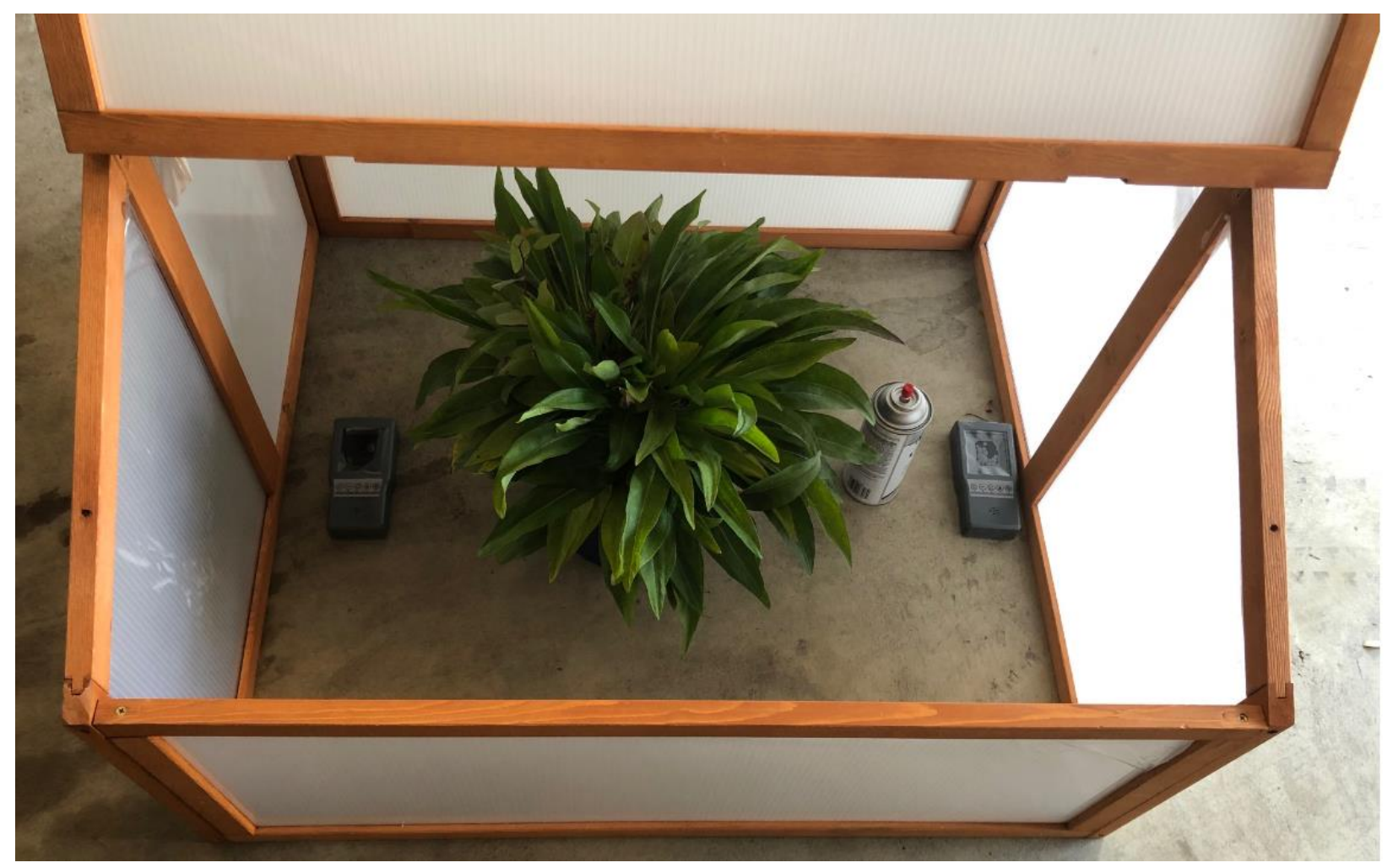

Figure 3. MCombo Double Box Wooden Greenhouse, with the Aster plant between the Air Quality Monitors, and spray to the right 


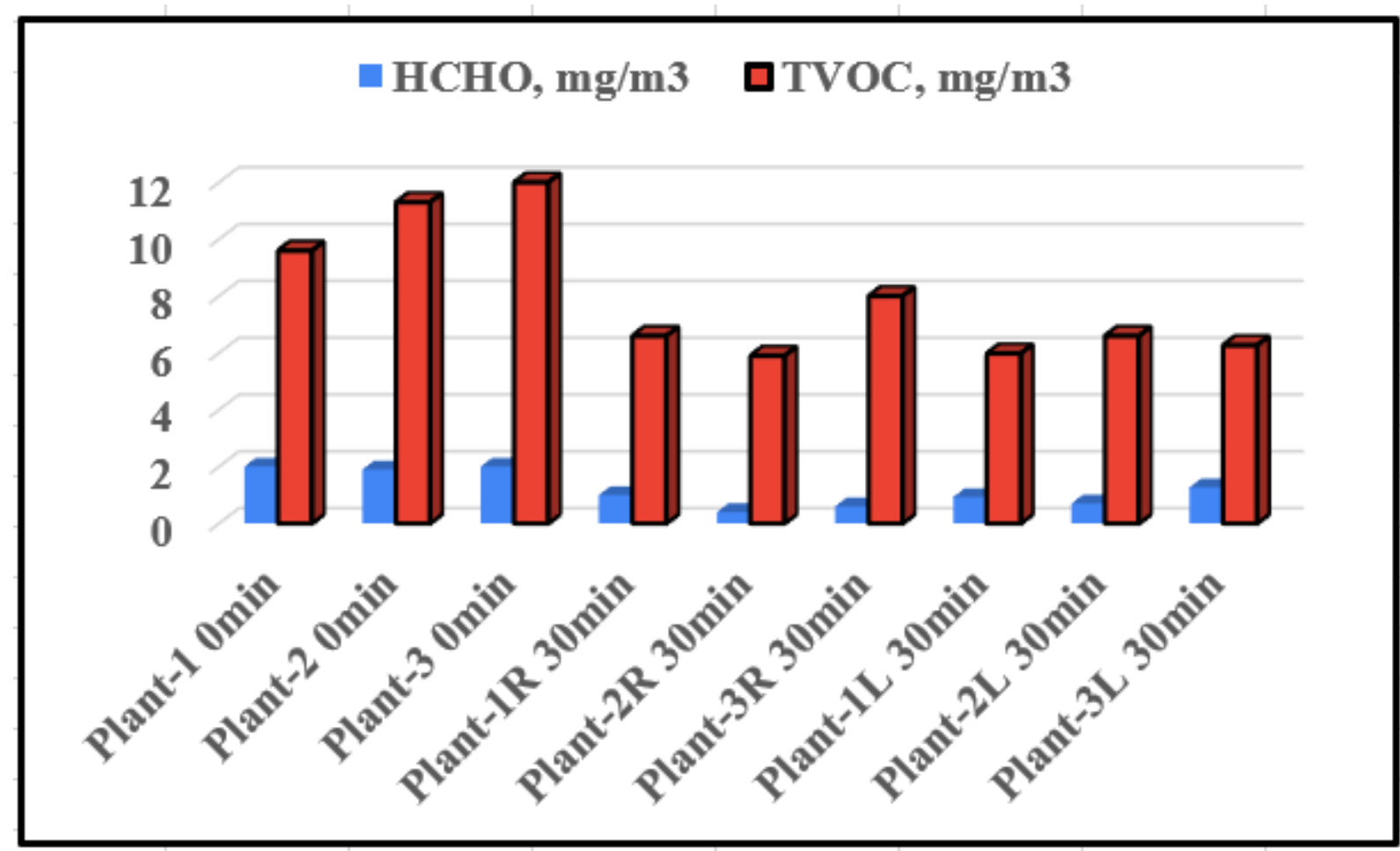

Figure 4. Measurements of Formaldehyde (HCHO), and Total Volatile Organic Compounds (TVOC) were taken after spraying them at 0 and 30 minutes using both monitors.

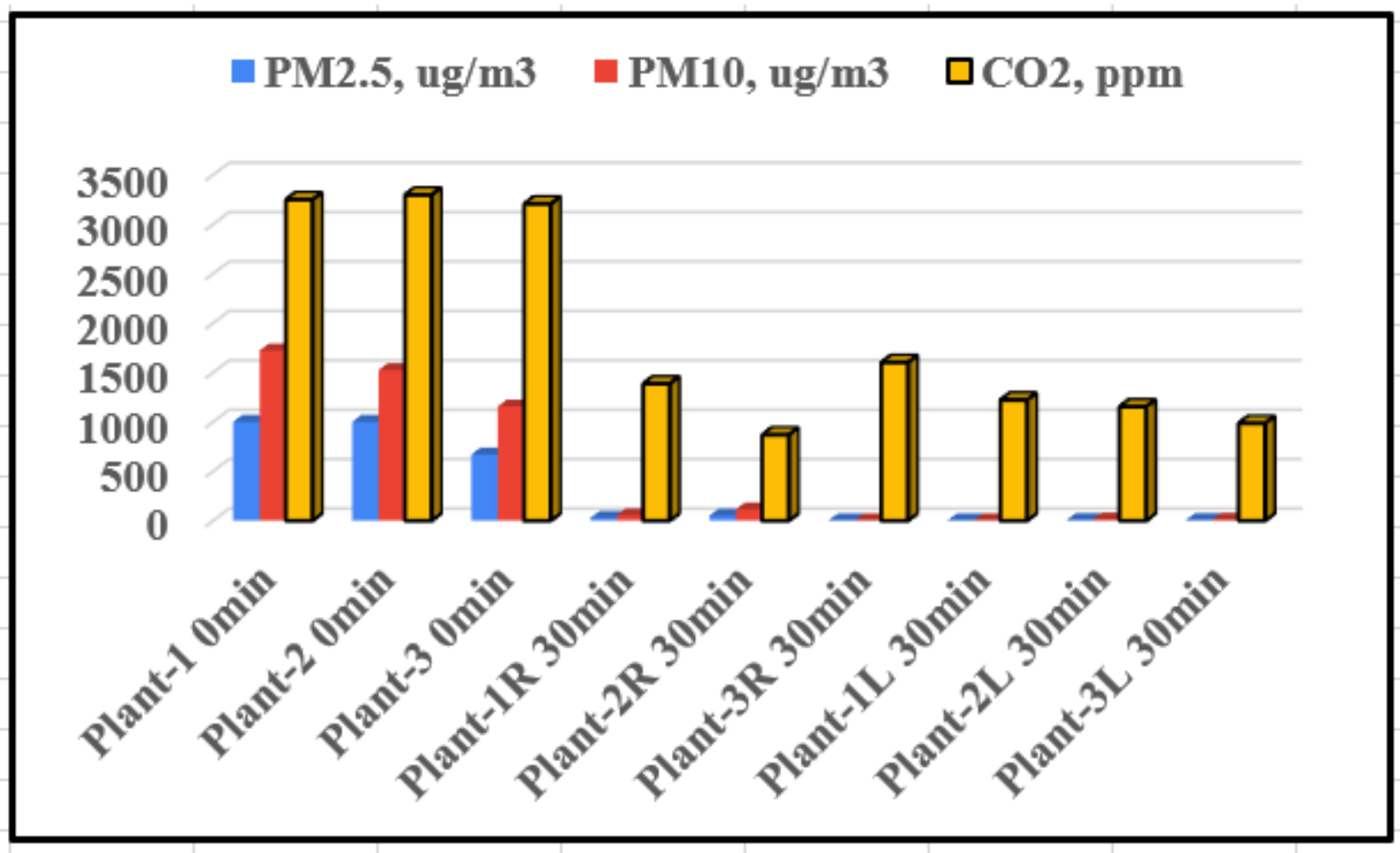


Figure 5. Measurements of carbon dioxide (CO2), and Particulate Matter (PM2.5/PM10) were taken after spraying them at 0 and 30 minutes using both monitors. 